\title{
Survival Probability at the LHC
}

\author{
Errol Gotsman \\ Department of Particle Physics, School of Physics and Astronomy, Raymond and Beverly Sackler \\ Faculty of Exact Science, Tel Aviv University, Tel Aviv 69978, Israel
}

\begin{abstract}
Using a model based on two elements: the Good-Walker mechanism for low mass diffraction and multi-pomeron interactions for high mass diffraction, we obtain an excellent description of all aspects of soft scattering at high energy. The parameters of the model are determined by a fit to experimental data, giving the slope of the pomeron to be $\alpha_{\mathbb{P}}^{\prime} \approx 0.01 \mathrm{GeV}^{-2}$. We calculate the survival probability of diffractive Higgs production, and obtained a value for this observable, which is smaller than $1 \%$ for the LHC energy range.
\end{abstract}

\section{Introduction}

This talk is based on the contents of a recent paper by Gotsman, Levin, Maor and Miller [1].

One of the key issues facing the high energy community is, whether the cornerstone of the Standard Model i.e. the Higgs boson will be discovered at the LHC. A suggested promising channel for its discovery is the diffractive process $p+p \rightarrow p+H+p$, with rapidity gaps between the final protons and the Higgs boson.

We are interested in obtaining a reliable estimate of the probability of seeing the Higgs at the LHC [1]. The detection of the Higgs boson in the above channel depends crucially on the survival probability of the rapidity gap [2], [3], a calculation for which one requires knowledge of:

- the "hard" amplitude for Higgs production, which is a short distance process, and can be calculated using PQCD.

- the survival factor of the gap also depends on the "soft" elastic amplitude, which is believed to be a long distance process, and has to be evaluated using a model describing the relevant "soft" p-p interactions.

We determine the parameters of the soft amplitudes by making a fit to a data base containing all the relevant published data on $p-p$ and $\bar{p}$-p interactions (see [1] for details). An unexpected result of our fit is that the value of the pomeron slope $\alpha_{I P}^{\prime}=0.012 \mathrm{GeV}^{-2}$. This is consistent with the assumption of [4], who take $\alpha_{\mathbb{P}}^{\prime}=0$. Our result suggests that the typical parton momentum is large (approximately $<p_{t}>=1 / \sqrt{\alpha_{\mathbb{P}}^{\prime}} \geq 7 \mathrm{GeV}$ ). Therefore, the running QCD coupling $\alpha_{I P}^{\prime}=\pi / b \ln \left(<p_{t}^{2}>/ \Lambda_{Q C D}^{2}\right) \ll 1$ (approximately 0.18 ), and we can consider it as our small parameter, when applying perturbative QCD estimates to the pomeron-pomeron interaction vertices.

The details of the fit as well as the values of the parameters are given in [1]. 


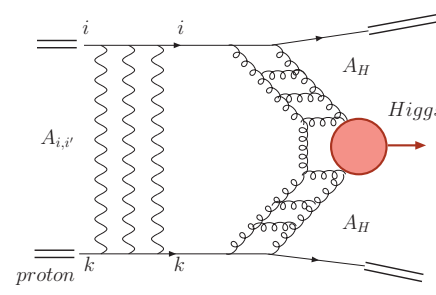

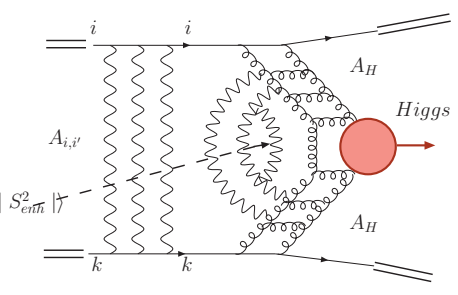

b)

FIGURE 1. Survival probability for exclusive central diffractive production of the Higgs boson. (a) shows the contribution to the survival probability in the G-W mechanism, while (b) illustrates the origin of the additional factor $\left\langle\left|S_{e n h}^{2}\right|\right\rangle$.

(1) In order to fit the single diffractive, double diffractive as well as the elastic amplitudes it is essential to include triple $\mathbb{P}$ diagrams (for the large mass diffractive contribution) in addition to the usual Good-Walker (G-W) mechanism (which account only for the low mass diffractive contribution).

(2) The fact that $\alpha_{\mathbb{P}}^{\prime}$ is so small, encourages us to use PQCD as a guide for building a theory of pomeron interactions valid for all distances, and there is no need for separate "soft" and "hard" pomerons.

(3) In the lowest order approximation of PQCD, only the transitions $\mathbb{P} \rightarrow 2 \mathbb{P}$ and $2 \mathbb{P} \rightarrow \mathbb{P}$, should be considered, as all other vertices are small. We, therefore restrict the summing of $\mathbb{P}$ diagrams to those with three $\mathbb{P}$ vertices only (i.e. fan diagrams).

(4) Since $4 \alpha_{\mathbb{I P}}^{\prime} \ln \left(s / s_{0}\right) \ll 1$ over the entire kinematic range, to simplify the algebra we take $\alpha_{I P}^{\prime}=0$.

(5) Details for summing the diagrams containing multiple pomeron (enchanced) interactions, and obtaining analytic expressions for the scattering amplitudes is given in [1].

\section{Survival Probability of Diffractive Higgs Production}

In the following we limit our discussion to the survival probability of Higgs production, in an exclusive central diffractive process. Most estimates of the values of survival probability have been made in the framework of G-W mechanism, in two channel eikonal models. A general review of such survival probability calculations can be found in [5]. The structure of the survival probability expression is shown in Fig.1a, i.e.

$$
\left\langle\left|S_{2 c h}^{2}\right|\right\rangle=\frac{N(s)}{D(s)},
$$

where

$$
\begin{gathered}
N(s)=\int d^{2} b_{1} d^{2} b_{2}\left[\sum_{i, k}<p\left|i>^{2}<p\right| k>^{2} A_{H}^{i}\left(s, b_{1}\right) A_{H}^{k}\left(s, b_{2}\right)\left(1-A_{S}^{i, k}\right)\right]^{2}, \\
D(s)=\int d^{2} b_{1} d^{2} b_{2}\left[\sum_{i, k}<p\left|i>^{2}<p\right| k>^{2} A_{H}^{i}\left(s, b_{1}\right) A_{H}^{k}\left(s, b_{2}\right)\right]^{2} .
\end{gathered}
$$

$<p \mid i>$ is equal to $\left\langle\Psi_{\text {proton }} \mid \Psi_{i}\right\rangle$ hence, $<p|1\rangle=\alpha$ and $<p|2\rangle=\beta$. $A_{S}$ denotes the 


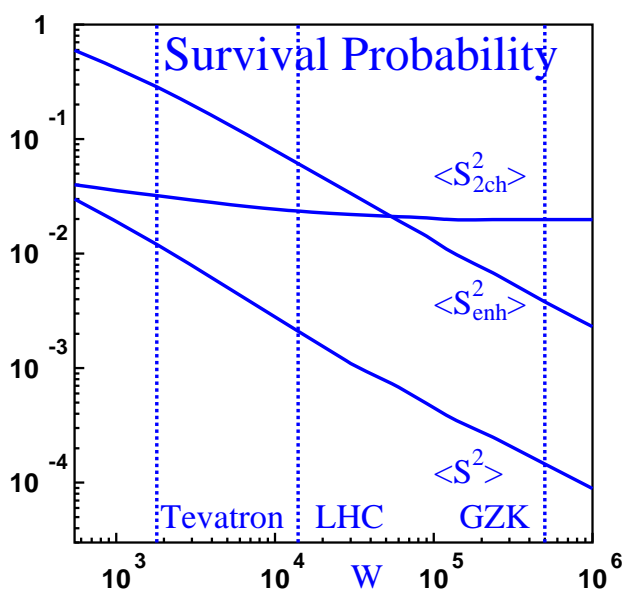

FIGURE 2. Energy dependence of the survival probability for centrally produced Higgs.

soft strong interaction amplitude.

The form of $A_{H}(s, b)$ has been discussed in Refs. [5, 6]. In our model we assume an input Gaussian $b$ dependence for the hard amplitudes. We have

$$
A_{i, k}^{H}=A_{H}(s) \Gamma_{i, k}^{H}(b),
$$

where $A_{H}(s)$ is an $s$ - dependent arbitrary function which does not depend on $i, k$, and $\Gamma_{i, k}^{H}(b)=\frac{1}{\pi\left(R_{i, k}^{H}\right)^{2}} e^{-\frac{2 b^{2}}{\left(R_{i, k}^{H}\right)^{2}}}$. The hard vertices and radii $R_{i, k}^{H}$, are constants derived from HERA $J / \Psi$ elastic and inelastic photo and DIS production[7].

Following Refs. [5, 8] we have introduced in the above, two hard $b$-profiles

$$
A_{H}^{p p}(b)=\frac{V_{p \rightarrow p}}{2 \pi B_{e l}^{H}} \exp \left(-\frac{b^{2}}{2 B_{e l}^{H}}\right) ; \quad A_{H}^{p d}(b)=\frac{V_{p \rightarrow d}}{2 \pi B_{i n}^{H}} \exp \left(-\frac{b^{2}}{2 B_{i n}^{H}}\right) .
$$

The values $B_{e l}^{H}=3.6 \mathrm{GeV}^{-2}$ and $B_{i n}^{H}=1 \mathrm{GeV}^{-2}$, have been taken from the experimental ZEUS data on $J / \Psi$ production at HERA (see Refs.[5, 9]).

Eqn.(1) does not give a correct estimate for the survival probability and should be multiplied by a factor $\left(\left\langle\left|S_{\text {enh }}^{2}\right|\right\rangle\right)$, which incorporates the possibility for the Higgs boson to be emitted from the enhanced diagrams (see fig1.b). Therefore, the resulting survival probability should be written as

$$
\left\langle\left|S^{2}\right|\right\rangle=\left\langle\left|S_{e n h}^{2}\right|\right\rangle \times\left\langle\left|S_{2 c h}^{2}\right|\right\rangle
$$

The results for the energy dependence of $\left\langle\left|S^{2}\right|\right\rangle,\left\langle\left|S_{2 c h}^{2}\right|\right\rangle$, and $\left\langle\left|S_{\text {enh }}^{2}\right|\right\rangle$ are shown in Fig.2. 
TABLE 1. Comparison of results of the GLMM [1] and KMR [4] models.

\begin{tabular}{||l|ll|ll|ll||}
\hline & \multicolumn{2}{|c|}{ Tevatron } & \multicolumn{2}{|c|}{ LHC } & \multicolumn{2}{|c}{$W=10^{5} \mathrm{GeV}$} \\
& GLM & KMR & GLM & KMR & GLM & KMR \\
\hline$\| S_{2 c h}^{2}(\%)$ & 3.2 & $2.7-4.8$ & 2.35 & $1.2-3.2$ & 2.0 & $0.9-2.5 \|$ \\
\hline$\| S_{\text {enh }}^{2}(\%)$ & 28.5 & 100 & 6.3 & 100 & 3.3 & 100 \\
\hline$\| S^{2}(\%)$ & 0.91 & $2.7-4.8$ & 0.15 & $1.2-3.2$ & 0.066 & $0.9-2.5 \|$ \\
\hline \hline
\end{tabular}

\section{Results, Discussion and Conclusions}

A paper by the Durham group (KMR) has recently been published [4], this paper has a similar philosophy to our paper [1], however the conclusions of the two papers regarding the values of the survival probability at the LHC, differ greatly.

The main differences are due to the fact that KMR:-

(1) Do not carry out a fit to determine the values of their parameters, they prefer to "fine tune" these, and do not quote a $\chi^{2} / d . f$. for their model.

(2) Use an adhoc assumption regarding the form of the multi-pomeron coupling i.e. they assume that $\Gamma[n(\mathbb{P}) \rightarrow m(\mathbb{P})]=m n \lambda^{m+n-2}$, where $\lambda$ is related to the difference between the absorptive cross section for an intermediate parton and that for the incoming proton. The above relation used for multi-pomeron coupling, has its origin in the pomeron calculus of the 1970's, and its validity in the present context is questionable.

(3) Assume $S_{\text {enh }}^{2} \approx 1$, (without any justification), and therefore their results for $S^{2}=S_{2 \text { channel }}^{2} \times S_{\text {enh }}^{2}$, are much larger than ours.

Table 1 contains a comparison of some of the results obtained in the two different approaches.

Bartels et al. [10] have also evaluated the gap survival probability (including the first pomeron loop) in the framework of pQCD, our results for $S_{\text {enh }}^{2}$ and $S_{2 c h}^{2}$ are very close to the values quoted in their paper, in spite of the very different formalism used. Consequently, Miller [11] evaluated the hard $\left\langle\left|S^{2}\right|\right\rangle$, (including the first enhanced diagram) for the BFKL pomeron, and obtained a value of $0.4 \%$.

We eagerly await results from the LHC, our calculations cast doubt that the Higgs will be discovered in the channel $p+p \rightarrow p+H+p$.

\section{Acknowledgements}

This research was supported in part by the Israel Science Foundation, founded by the Israeli Academy of Science and Humanities, by BSF grant \# 20004019 and by a grant from Israel Ministry of Science, Culture and Sport and the Foundation for Basic Research of the Russian Federation.

\section{REFERENCES}

1. E. Gotsman, E. Levin, U. Maor and J. S. Miller, Eur.Phys.J.C, (in print) arXiv:0805.2799 hep-ph].

2. J. D. Bjorken, Int. J. Mod. Phys. A7,4189 (1992); Phys.Rev. D47,101 (1993). 
3. E. Gotsman, E. Levin and U. Maor, Phys.Rev. D49,R4321 (1994).

4. M. G. Ryskin, A. D. Martin and V. A. Khoze, Eur.Phys.J. C54, 199 (2008).

5. E. Gotsman, E. Levin, U. Maor, E. Naftali, A. Prygarin, in HERA and the LHC: A workshop on the Implications of HERA for LHC Physics: Proceedings Part A,221(2005).

6. E. Gotsman, E. Levin and U. Maor, Phys.Rev. D60, 094011 (1999).

7. Zeus Collaboration: Nucl.Phys. B695, 3 (2004): Eur.Phys.J. C24, 345 (2004).

8. E. Gotsman, A. Kormilitzin, E. Levin and U. Maor, Eur.Phys.J., C52,295 (2007).

9. H. Kowalski, D. Teaney, Phys.Rev. D68,114005 (2003).

10. J. Bartels, S. Bondarenko, K. Kutak and L. Motyka, Phys.Rev. D73, 093004 (2006).

11. J.S. Miller, Eur.Phys.J., C56,39 (2008). 